\title{
Finite element method application to calculate the friction discs of the hydromechanical transmission control system
}

\author{
Alexey Khomichev ${ }^{1, *}$, and Ilya Trusevich $^{2}$ \\ ${ }^{1}$ Kurgan state university, Faculty of Transport Systems, Department of Tracked Vehicles and Applied \\ Mechanics, 640020, Kurgan, Russia \\ ${ }^{2}$ IES UB RAS, Department of Transportation Vehicles Mechanics, 620049, Ekaterinburg, Russia
}

\begin{abstract}
In article presents the results of experimental researches of friction elements of a hydromechanical transmission control system, the loadings to which they are subjected. Substantiates the need to improve the methodology for calculating friction discs.
\end{abstract}

Modern automatic transmission with electrohydraulic control can significantly improve the comfort of control reduce fuel consumption through the selection of the optimal control law of the gearshift. The tests have shown that the transmission parts are subjected to significant dynamic loads limiting their durability. In particular, the increased load is experienced by the metal-ceramic discs of the friction control units of the gearshift [1].

Wear and deformation are typical causes of friction discs failure. However, it was noticed a new kind of failure - a disruption discs, which is observed in the developed transmissions as well as in designs world leader in the design and production of transmissions - Allison company (Fig. 1).

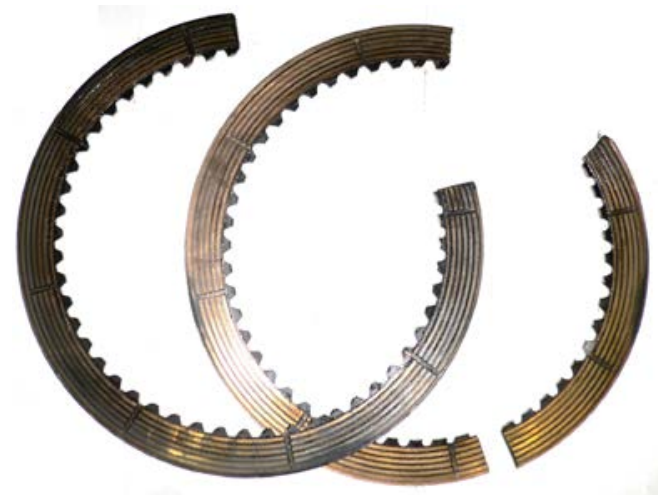

Fig. 1. Destruction of metal-ceramic discs

* Corresponding author: khomichev.alexey@mail.ru 
This phenomenon occurs when the torque converter operates in the mode of hydraulic coupling with a slight slip of the turbine wheel relative to the pump wheel. Engine and gears cannot create harmonics of such a high order. Therefore, the hypothesis of new dynamic phenomenon - excitation of resonant oscillations by a torque converter - was put forward.

The hypothesis about the failure of metal-ceramic discs due to the coincidence of their natural frequency with the frequency generated by the torque converter is based on the nature of the spectral density. In the pre-locking mode of the torque converter the spectral density graph has a pronounced amplitude surge. After locking of the torque converter bursts of amplitude are absent that is resonant modes are impossible (Fig. 2).

Torque converter is unlocked

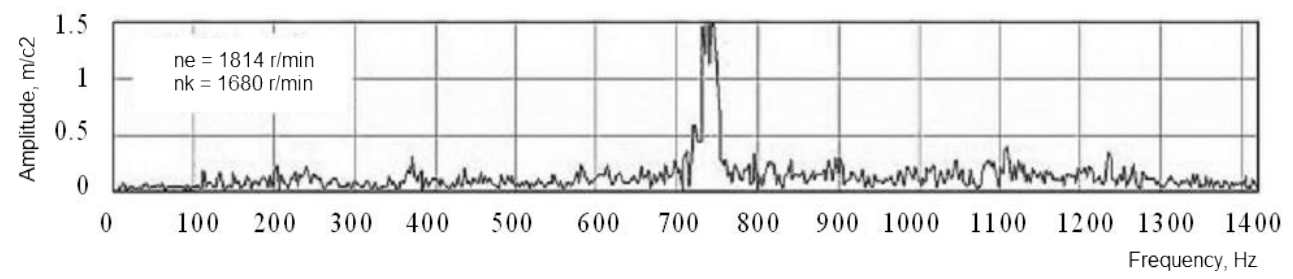

Torque converter is locked

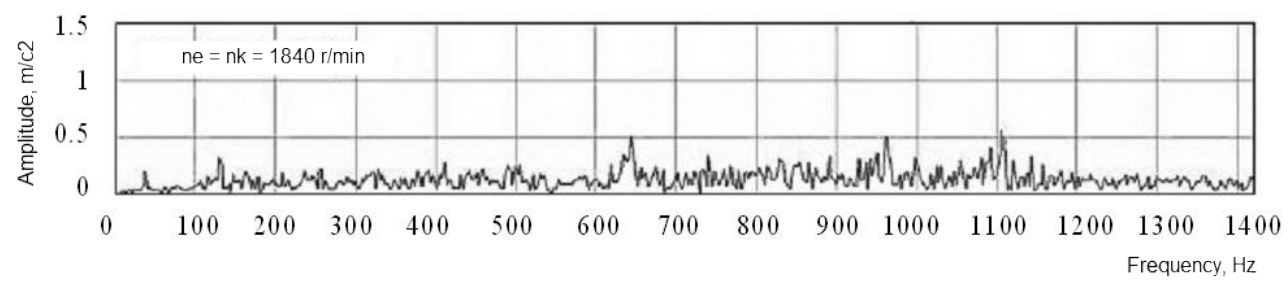

Fig. 2. Spectral density.

Current calculation methods of the friction control units allow to determine the heat release rate of metal-ceramic discs in the process of slipping, the maximum speed of their rotation on the condition of strength and durability. However, the actual operating modes differ significantly from the calculated ones.

To solve the problem of prediction of vibration loading of the metal-ceramic discs it is possible to use the approximate analytical dependence. The disc consists of a steel ring of rectangular cross section with the ring gear and a metal-ceramic friction linings which are welded to the steel ring through the adhesive layer. Analytically determine the natural frequency of the disc is quite difficult due to differences in physical and mechanical properties of materials. Therefore, we define the natural frequency of the steel ring with known geometric parameters.

The natural frequency of the ring is determined according to the wave theory [2]. Design of steel ring conforms to the main assumptions taken in studies of the circular rings. In this case, it is considered as a circular bar of bounded curvature with a constant crosssection (Fig. 3).

There are two cases for bending vibrations of a circular ring: bending vibrations in the plane of the ring and bending vibrations, including both moving perpendicular to the plane of the ring and torsion. The process of oscillation of the ring can be described by a system of hyperbolic partial differential equations, which, when considering the oscillations of bending in the plane of the ring, with the exception of the coordinates, is reduced to a 
differential equation of sixth order. The equation describes the tangential displacement $x_{2}$ of the cross section of the ring defined by the corner coordinates $\varphi$ :

$$
\frac{\partial^{6} x_{2}}{\partial \varphi^{6}}+2 \frac{\partial^{4} x_{2}}{\partial \varphi^{4}}+\frac{\partial^{2} x_{2}}{\partial \varphi^{2}}+\frac{m_{0} R^{4}}{E I} \cdot \frac{\partial^{2}}{\partial^{2}}\left(\frac{\partial^{2} x_{2}}{\partial \varphi^{2}}-x_{2}\right)=0
$$

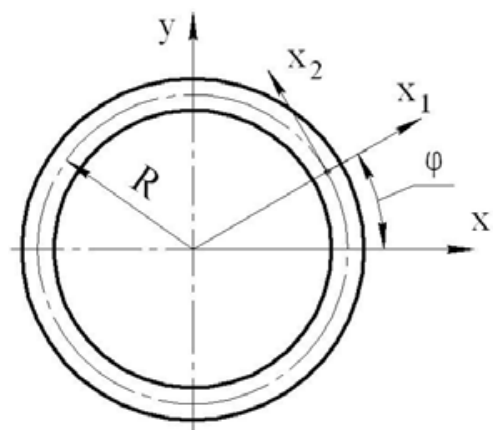

Fig. 3. Calculation scheme for determining the natural frequency of the ring

The solution of the equation is sought in the form of radial $x_{1}$ and tangential $x_{2}$ displacements

$$
\begin{aligned}
& x_{1}=U_{1}(\varphi) \cdot \cos (\omega \cdot t) \\
& x_{2}=U_{2}(\varphi) \cdot \sin (\omega \cdot t)
\end{aligned}
$$

At the same time, an ordinary differential equation is obtained for the $U_{2}$ :

$$
\left\{\begin{array}{c}
\frac{d^{6} U_{2}}{d \varphi^{6}}+2 \frac{d^{4} U_{2}}{d \varphi^{4}}+\frac{d^{2} U_{2}}{d \varphi^{2}}-\frac{m_{0} R^{4} \omega^{2}}{E I}\left(\frac{d^{2} U_{2}}{d \varphi^{2}}-U_{2}\right)=0 \\
U_{1}=-\frac{d U_{2}}{d \varphi}
\end{array}\right.
$$

According to the general rules for solving differential equations, it is necessary to find a general solution of the equation, including six constants, and subordinate it to the boundary conditions. The zero equality of the determinant of the system expressing the boundary conditions leads to the frequency equation.

For a ring, the boundary conditions are replaced by periodicity conditions that are satisfied if

$$
\begin{aligned}
& U_{1}=K \cdot \sin (K \cdot \varphi) \\
& U_{2}=\cos (K \cdot \varphi)
\end{aligned}
$$

Substituting (5), (6) in (4), we establish that the latter is satisfied with the same, if the frequency is

$$
\omega=\omega_{k}=\frac{K\left(K^{2}-1\right)}{\sqrt{K^{2}+1}} \sqrt{\frac{E I}{m_{0} R^{4}}}
$$


where $K$ an integer that determines the number of waves that fit the length of the ring, $E$ is Young modulus, $I$ is the moment of inertia of the ring about the axis $x, b$ is the width of the cross section of the disc, $h$ is the thickness of the disc, $m_{0}$ is the weight of the ring, $R$ is average radius of the ring.

In order to solve the problem of prediction of vibration loading of metal-ceramic discs with a high degree of accuracy and to obtain more reliable results it is advisable to use the finite element method [3]. This method is a numerical method for solving differential equations in physics and engineering problems [4-7].

The peculiarity of the method is that it requires cumbersome calculations even when solving very simple problems. However, due to the use of high-speed computers and modern software, calculations do not take much time and the results are quite accurate. One of these software is COSMOSWorks [8].

In solving the problems of determining the natural frequencies and waveforms, there are limitations: damping, assembly kinematics and friction are ignored. As a result, it is impossible to obtain information about the state of the structure at the time of resonance. Displacements, deformations, stresses are not calculated. The reason is that all of these parameters theoretically have an infinite value. It is also necessary to carefully analyze problems with movable assemblies. They should be considered in two aspects: resonance of parts as isolated objects under idealized boundary conditions; resonance assembly and its functioning in this state. The frequencies of real mechanical systems are practically independent from the natural frequencies of parts in their calculation in the mode of dynamics.

However, even taking into account these limitations, the program allows you to solve the most urgent problem-to perform analysis of products and modify them to exclude from the range of unwanted natural frequencies and resonance.

The values of natural frequencies obtained in the calculation of a circular ring made of steel $\left(E=2.1 \cdot 10^{11} \mathrm{~Pa}, R=0.146 \mathrm{~m}, b=0.028 \mathrm{~m}, h=0.001 \mathrm{~m}\right)$ are shown in Table 1 . Some form diagrams of natural oscillations are shown in Fig. 4.

Table 1. Result of calculation the natural frequency of the ring

\begin{tabular}{|c|c|c|c|}
\hline \multirow{2}{*}{$\begin{array}{c}\text { Oscillation } \\
\text { form } \\
\text { number }\end{array}$} & Analytical dependence & Finite element analysis & \multirow{2}{*}{ Error, \% } \\
\cline { 2 - 3 } & 0 & 0 & 0 \\
\hline 1 & 0 & 0 & 0 \\
\hline 2 & 29.286 & 31.072 & 5.748 \\
\hline 3 & 82.833 & 86.162 & 3.684 \\
\hline 4 & 158.825 & 163.98 & 3.144 \\
\hline 5 & 256.854 & 264.33 & 2.828 \\
\hline 6 & 376.799 & 387.22 & 2.691 \\
\hline 7 & 517.067 & 532.64 & 2.633 \\
\hline 8 & 680.715 & 700.62 & 2.617 \\
\hline 9 & 866.212 & 891.12 & 2.618 \\
\hline 10 & 1073.549 & 1071.9 & 0.302 \\
\hline 11 & 1302.723 & 1339.4 & 2.619 \\
\hline 12 & 1553.731 & 1597.2 & 2.621 \\
\hline 13 & 1826.572 & 1877.1 & 2.606 \\
\hline 14 & 2121.244 & 2179.1 & 2.581 \\
\hline 15 & 2437.746 & 2502.9 & 2.539 \\
\hline 16 & 2776.079 & 2848.8 & 2.496 \\
\hline 17 & & & \\
\hline
\end{tabular}



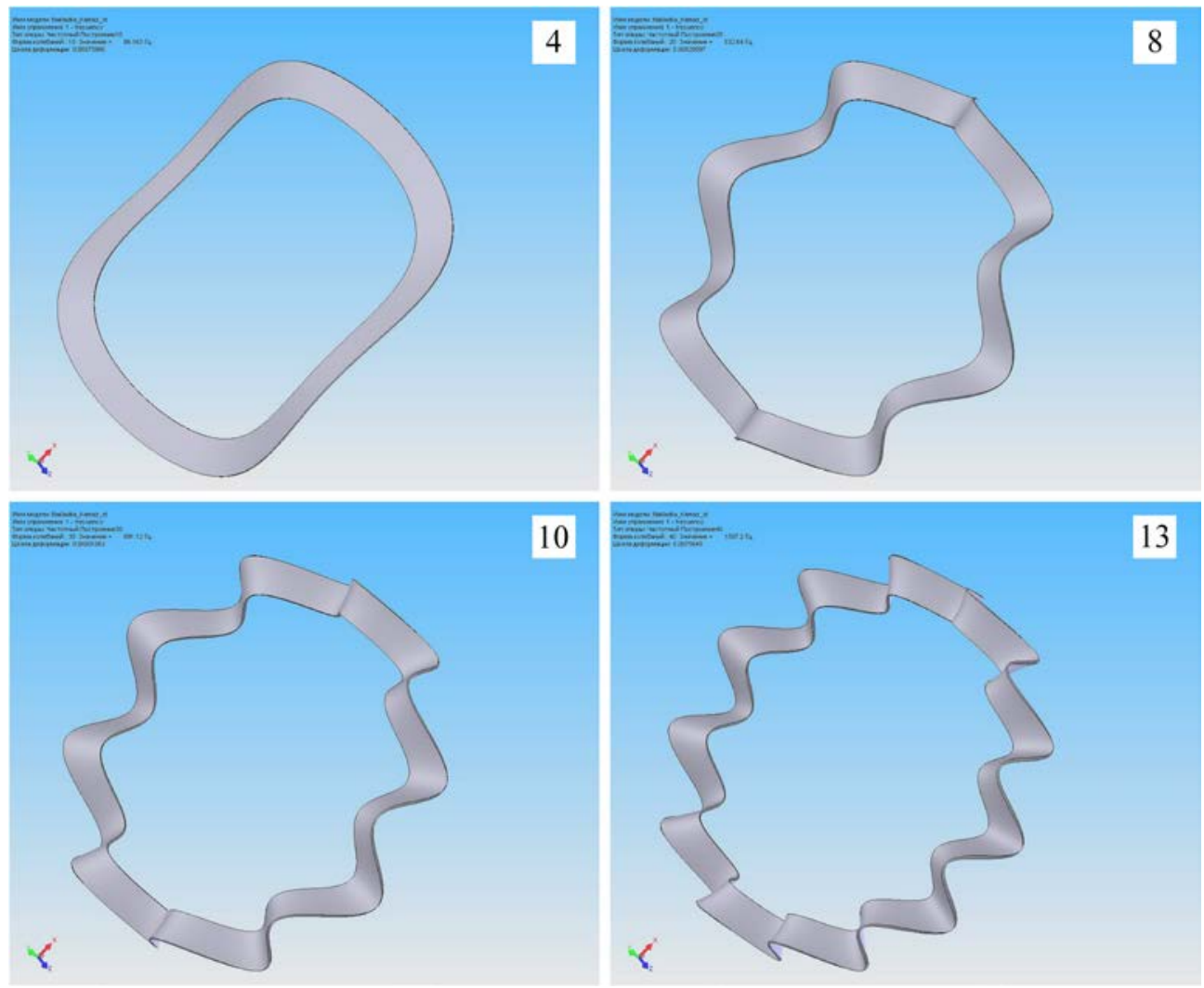

Fig. 4. Diagrams of natural oscillations of the ring

The obtained results allow to conclude that the proposed method of calculation is reliable and can be used to determine the natural frequencies and oscillation forms of metalceramic disks. This makes it possible already at the design stage to select the optimal design of disks and to exclude from the spectrum of the oscillations the undesirable values of natural frequencies. Thus, it is possible to avoid the negative effect of the resonant modes on the durability of the elements of the transmission control system.

This study was carried out with partial support under the state task IES UB RAS No. 0391-2014-007.

\section{References}

1. V.B. Derzhansky, I.A. Taratorkin, Dynamic load prediction for transport vehicles transmission. (Kurgan state university publishing, Kurgan, 2008)

2. S.P. Timoshenko, D.H. Young, W. Weaver, Vibrations in engineering. (Mashinostroenie, Moscow, 1985)

3. O.C. Zienkiewicz, R.L. Taylor, The finite element method: solid mechanics. Vol. 2 (Butterworth-heinemann, 2014)

4. M. Sága, R. Bendár, M. Vaško, Contribution to modal and spectral interval finite element analysis. Vibration Problems ICOVP 2011, Springer Processing in Physics 139, 269-274 (2011) 
5. M. Sága, M. Vaško, P. Kopas, W. Piekarska, T. Domański, M. Kubiak, Contribution to fatigue damage prediction of thin shell finite element methods. MATEC Web of Conferences 157, Machine Modeling and Simulations (2018)

6. M. Nad', R. Ďuriš, T. Nánási, Prediction of modal properties of circular disc with prestressed fields. MATEC Web of Conferences 157, Machine Modeling and Simulations (2018)

7. F. Kuratani, S. Yano, Vibration analysis of a circular disc tensioned by rolling using finite element method. Archive of Applied Mechanics 70, 279-288 (2000)

8. A.A. Alyamovskiy, SolidWorks/COSMOSWorks. Engineering FEM analysis (DMK Press, 432, 2004) 